\title{
A Randomized, Controlled Clinical Trial of Exercise in Patients with Spinal Muscular Atrophy: Methods and Baseline Characteristics
}

\author{
Jacqueline Montes ${ }^{\mathrm{a}, \mathrm{b}, *}$, Carol Ewing Garber ${ }^{\mathrm{c}}$, Samantha S. Kramer ${ }^{\mathrm{a}}$, Megan J. Montgomery ${ }^{\mathrm{a}}$, \\ Sally Dunaway ${ }^{\mathrm{a}}$, Shirit Kamil-Rosenberg ${ }^{\mathrm{c}}$, Brendan Carr ${ }^{\mathrm{c}}$, Nancy E. Strauss ${ }^{\mathrm{b}}$, Douglas Sproule ${ }^{\mathrm{a}}$ \\ and Darryl C. De Vivo ${ }^{\mathrm{a}}$ \\ ${ }^{a}$ Department of Neurology, Columbia University Medical Center, NY, USA \\ ${ }^{\mathrm{b}}$ Department of Rehabilitation and Regenerative Medicine, Columbia University Medical Center, NY, USA \\ ${ }^{\mathrm{c} B i o b e h a v i o r a l ~ S c i e n c e s}$ Department, Teachers College, Columbia University, NY, USA
}

\begin{abstract}
.
Background: Spinal Muscular Atrophy (SMA) is a recessively-inherited neuromuscular disease characterized by weakness and muscle atrophy. Although anecdotal benefits from exercise have been noted, and despite promising pre-clinical and pilot reports, the effect of exercise has not been addressed in a controlled trial in SMA.

Objective: To assess the effects of exercise on measures of function, strength, and exercise capacity in ambulatory SMA patients. Methods/Design: An evaluator-blinded, randomized, controlled trial of aerobic and strengthening exercise in 14 ambulatory SMA patients aged 8-50 years. Patients will be randomized to either the exercise or control arm after the 1 month lead in period. During the first 6-months, the exercise group will receive the intervention while the other group serves as a control. After those 6 months, both groups will receive the intervention. The last 6-months of the study are designed to mimic real-world conditions where all participants are encouraged to continue on their own. Participants will be monitored throughout this 19 month study and will have in-person visits every three months. The primary outcome measure is the change in the total distance walked over 6-months on the six minute walk test (6MWT). Secondary outcome measures include maximal oxygen uptake (VO2 max), functional and strength assessments, pulmonary function, fatigue, and quality of life.

Discussion: The result of this prospective, single blinded, randomized and controlled clinical trial of exercise on an established functional outcome measure will have impact on clinical practice by providing important guidance to clinical management of SMA patients.
\end{abstract}

Keywords: spinal muscular atrophy, exercise, six minute walk test, strength

\section{INTRODUCTION}

Spinal Muscular Atrophy (SMA) is a progressive, recessively-inherited neuromuscular disease

\footnotetext{
${ }^{*}$ Correspondence to: Dr. Jacqueline Montes, SMA Clinical Research Center, Department of Neurology, Columbia University, 180 Ft. Washington Avenue, 5th Floor, New York, NY 10032, USA. Tel.: +1 212342 5767; Fax: +1 212305 9320; E-mail: jm598@ columbia.edu.
}

characterized by weakness and muscle atrophy due to the loss of spinal cord motor neurons. It is an autosomal recessive disorder genetically characterized by the absence of the Survival Motor Neuron (SMN1) gene located on chromosome 5q13 [1]. Despite identification of a single mutation, SMA is phenotypically heterogeneous ranging from a life threatening to life altering disease [2]. Individuals with SMA in its mildest form, type 3, are an ideal target population for initial studies of exercise in SMA because 
their relatively preserved residual strength makes both endurance and strengthening programs feasible.

SMA type 3 patients have proximal weakness and impaired ambulation, yet are able to walk unaided for at least some period of their lives. Many SMA type 3 patients, however, ultimately lose the ability to walk independently, possibly due in part to deconditioning from physical inactivity. While symptom onset after three years of age is associated with a greater probability of maintaining independent ambulation [3], the role of modifiable risk factors and interventions such as exercise or endurance training on motor function has not been well addressed.

Among healthy individuals and individuals with disabling conditions, exercise has been shown to improve cardiorespiratory endurance capacity, muscle strength, power and endurance in children and adults [4-6], and physical and motor function in older adults and children $[5,7,8]$ through numerous physiologic changes including improved muscle histology and architecture, muscle oxidative and glycolytic capacity, and motor and cardiorespiratory function [5, 9]. Furthermore, exercise training can reverse muscle atrophy and decrements in muscle strength resulting from prolonged inactivity $[10,11]$. Exercise results in acute and chronic changes in muscle mitochondria, potentially through increased mitochondrial DNA (mtDNA) and mRNA expression, or through upregulation of individual mitochondrial enzyme complexes of the electron transport chain [12, 13].

In other neuromuscular disorders, there are several examples of the beneficial effects of exercise programs on aerobic capacity and strength. In mitochondrial myopathies, exercise training improved endurance, peak capacity of work and quality of life.[14] Aerobic training in Becker muscular dystrophy [15], McArdle disease [16], myotonic dystrophy, facioscapulohumeral muscular dystrophy [17], limb girdle muscular dystrophy [18], and inclusion body myositis [19] have shown positive effects on exercise capacity. However, none of these studies were randomized or included a disease control group for comparison. In a recent meta-analysis of two controlled and three randomized clinical trials in muscular dystrophy, there was no evidence of benefit in muscle strength or endurance however the direction of effects favored exercise [20]. An individualized, progressive, resistive strengthening program improved function and quality of life in patients in a randomized controlled study of patients with adult motor neuron disease [21]. However, a home-based endurance exercise program of similar intensity among a heterogeneous group of patients with neuromuscular disorders showed no benefit in measures of function despite increases in leg strength [22].

Preclinical studies also suggest a beneficial effect of exercise in SMA. In a randomized trial of exercise in transgenic SMA mice, animals treated with a runningbased training program had significantly increased survival, delayed muscle atrophy, and preserved muscle fiber morphology [23]. In another preclinical study, exercise not only delayed motor neuron death, but also increased the postnatal maturation rate of transgenic SMA mice motor units [24].

While there have been no published studies on aerobic conditioning in SMA patients, strength training may yield some beneficial effects. In a pilot study of 6 SMA type 3 patients who underwent twelve sessions of a unilateral isokinetic leg exercise training, there was an increase in leg muscle strength by $33 \%$ in children and up to $122 \%$ in adults, compared to a maximum of $17 \%$ increase in the untrained leg [25]. Similarly, a case study of a young child with SMA type 3 described improvements in gross motor function and gait after an individualized aquatic therapy program [26].

The few reports that have been published have demonstrated no adverse effects associated with exercise in neuromuscular disease, supporting the safety of the intervention in this population [27]. However, the regimens employed in many of these studies were mild or conservative in intensity and volume, and thus were unlikely to be of adequate dose to have a measureable or noticeable clinical effect. This conservative approach mirrors clinical practice in neuromuscular disease, but it does not meet the physical activity guidelines recommended for healthy individuals [4]. Importantly, there is little evidence to suggest high intensity exercise is harmful in denervating disorders. Even in disorders where the pathology originates at the muscle level, responses to maximal strength training is similar to healthy individuals without residual damage [28]. To study the safety and efficacy of an individualized moderately intensive strengthening and endurance regime in SMA, a controlled trial is needed.

However, there is little evidence to guide clinicians as to what would comprise the optimal exercise regimen in terms of the type, intensity, and frequency of exercise for SMA patients. The effect of exercise on clinical measures of strength and function would provide mechanistic information on the changes underlying any observed improvement in exercise performance. These results would also inform our understanding of the mechanisms underlying weakness and fatigue in SMA. 


\section{METHODS}

\section{Study design}

A single-center, evaluator-blinded, randomized, controlled clinical trial (RCT) will be conducted to evaluate the effects of aerobic and strengthening exercise on motor function and strength in 14 patients with SMA. All participants will undergo one month of initial observation without treatment, following enrollment and randomization. This lead in period will allow for two baseline assessments of outcomes measures as well as provide time to create an individualized exercise plan tailored to the participants' baseline strength and exercise capacity measurements. Starting after month 1, group 1 will receive 6 months of intervention, followed by an additional 6 months of closely monitored ongoing treatment while maintaining evaluator blinding, followed by another 6 months of participantdirected exercise. Group 1, the participants receiving the intervention during the initial 6-months of the study, will serve as the experimental arm. Group 2 will serve as a wait-list control group. This cohort will receive 6 months of exercise intervention starting in the 7 th month. For the final 6 months of the study (months 13 to 19), both cohorts will be encouraged to maintain the exercise intervention on their own and instructed to continue to record efforts in an exercise diary to mimic "real world" conditions (Fig. 1).

To maintain the blind, study personnel as well as patients and families will be instructed not to discuss study design, group assignment, or exercise program with the primary evaluator. This blinded evaluator will not be permitted to review the study chart. She will be provided with the forms necessary for evaluation in a separate binder. If group assignment is unintentionally revealed to the blinded evaluator, it will be documented in the participant's study chart.

The study protocol has been approved by the Columbia University Institutional Review Board and the USAMRMC Human Research Protection Office. Informed consent will be obtained from all participants and parents for children under age 18 years. An independent safety monitor will evaluate participant data regularly for any adverse effects of the intervention.

\section{Study population}

The aim is to recruit 14 ambulatory patients between the ages of 8 and 50 years with genetically confirmed SMA. Patients will be recruited from existing population at the SMA Clinical Research Center at Columbia University Medical Center, the Pediatric Neuromuscular Clinical Research Network, and ClinicalTrials.gov

(a)

Study Timeline

Month

0

4

10

13

19

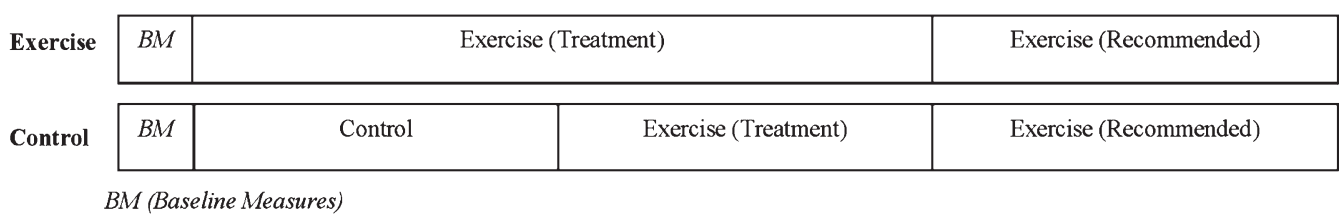

(b)

Video Conference

Month

$\begin{array}{llllllllllllllllllll}0 & 1 & 2 & 3 & 4 & 5 & 6 & 7 & 8 & 9 & 10 & 11 & 12 & 13 & 14 & 15 & 16 & 17 & 18 & 19\end{array}$

Exercise

\begin{tabular}{|l|l|l|l|}
\hline$\underset{8}{\sum_{0}}$ & $\begin{array}{l}\text { Bi- } \\
\text { weekly }\end{array}$ & Monthly & $\begin{array}{l}\text { Communication as Needed } \\
\text { (Participant Initiated) }\end{array}$ \\
\hline
\end{tabular}

Control

\begin{tabular}{|l|r|l|l|l|}
\hline $\begin{array}{l}\text { Communication as Needed } \\
\text { (Participant Initiated) }\end{array}$ & $\begin{array}{l}\sum \\
\&\end{array}$ & $\begin{array}{l}\text { Bi- } \\
\text { weekly }\end{array}$ & Monthly & $\begin{array}{l}\text { Communication as Needed } \\
\text { (Participant Initiated) }\end{array}$ \\
\hline
\end{tabular}

Fig. 1. Study design and schedule of in-clinic (a) and video conference (b) visits. Assessments are performed during the 7 in-clinic visits. 
(NCT01166022). All patients who meet eligibility will be invited to participate. Participants must be able to walk at least 25 meters without assistance, tread the stationary cycle ergometer, and be in good health, based on the findings of the physical exam and the judgment of the clinical investigator at the time of the screening assessment. Patients will be excluded if they use investigational medications intended for the treatment of SMA, have a contraindication to exercise according the American College of Sports Medicine (ACSM) criteria $[29,30]$, or are pregnant or breastfeeding.

\section{Intervention - cycle and strengthening}

The exercise protocol will include a muscle strengthening program in combination with a home based recumbent cycle ergometry program consistent with existing recommendations for physical activity in healthy children and adults [4, 5]. We will use a recumbent cycle ergometer at moderate intensity based on their functional capacity (peak oxygen uptake; VO2peak) measured during the maximal exercise test. The aerobic exercise will be performed five times weekly with target duration of 30 minutes per day performed continuously in one session or in multiple shorter exercise bouts performed several times per day to accumulate 30 minutes per day. If the participant is unable to attain 30 minutes of exercise per day they will exercise for a duration that is comfortable, increasing the duration of the exercise sessions progressively over time with a goal to attain the target of 30 minutes of aerobic exercise per day. The participants will be instructed to maintain a moderate intensity of exercise using OMNI scale ${ }^{\mathrm{TM}}$ ratings of perceived exertion for cycling [31, 32], which corresponds to a score between 5 and 7 .

Individualized strengthening exercises will comprise all major muscle groups including those identified as most susceptible to weakness in SMA [33]. Strengthening exercises will be performed three times weekly for approximately 30 minutes. After formal assessment of baseline muscle strength, an individualized treatment protocol will be established to detail resistance and repetitions needed to achieve the desired treatment effect [34]. For weaker muscles that are unable to move the limb against gravity, isometric or modified exercises in a gravity-eliminated position will be prescribed. We will follow the recommendation of the ACSM by using rest intervals of 2-3 min between each set of repetitions [34]. To improve muscular strength, mass, and endurance, a resistance exercise that allows a person to complete 8 to 12 repetitions per set is our goal. This translates to a resistance that is $\sim 60 \%$ to $80 \%$ of the individual's one repetition maximum or the greatest amount of weight lifted for a single repetition. Similar to the cycling portion of the exercise protocol, intensity will be determined using the OMNI scale ${ }^{\mathrm{TM}}$ ratings for resistance exercise, which corresponds to a score between 5 and 7 .

Exercise intensity or duration can be increased either during an in-clinic or teleconferencing visit. Additionally, participants will be permitted to advance their program provided they maintain a submaximal level of intensity as measured on the OMNI scale of perceived exertion. Participants will be instructed not to increase the intensity of their exercise more than once in a 2 week period. Participants will be allowed to perform aerobic exercise (cycling) more than the targeted 30 minutes only if they maintain a moderate intensity. Alternatively, participants will be advised that once the target has been achieved and maintained, increasing the resistance on the recumbent bicycle over short intervals remains an option to advance their exercise program. Both intensity (resistance) and duration of the cycling exercise will be captured on the patient diary.

Compliance will be monitored by several methods. First, the participant will wear a Polar ${ }^{\mathrm{TM}}$ Heart Monitor, which will record heart rate while performing the in-home cycle exercise program. In addition, the participant will complete a patient diary recording the exercise performed, duration, OMNI rating and any symptoms, adverse events, and other comments. These diaries will be reviewed during teleconferencing visits and collected at in-person visits. Program compliance will be enhanced by the use of customized illustrated instructional exercise sheets provided to patients. To ensure that the subjects are performing the exercise correctly and to enhance exercise safety and compliance, we have added an internet video-conferencing component performed at regularly scheduled intervals. During the internet video-conferences, exercise compliance, adverse events and participant questions and concerns will be discussed with an un-blinded member of the study personnel. These encounters will occur weekly during the first month of exercise, every other week for the next 2 months, and then monthly for 3 months over the initial 6 month intervention period. Communication between in-clinic visits in the subsequent 6 months of the exercise intervention and for the control group during the initial 6 months is encouraged as needed although no formal visits will be scheduled. All participants will be instructed to communicate with study intervention personnel as needed via text, phone, or email in the case of any questions or concerns. Spe- 
cific questions concerning any adverse events will be collected during every participant contact including all in-person and video-conferencing visits. Adverse events that will be queried for all participants include falls, excessive fatigue, muscle soreness, illnesses and other health related events.

\section{Outcome measures}

Several primary and secondary outcome measures will be collected, because these are variables that may be expected to change with exercise training or to monitor potential adverse effects of the training.

\section{Primary outcome measure - six minute walk test (6MWT)}

The primary outcome measure is the change from baseline to Month 6 in the total distance walked on the six minute walk test (6MWT). The 6MWT, an objective evaluation of functional exercise capacity, measures the maximum distance a person can walk in six minutes over a 25-meter linear course. Distance walked over the entire 6 minute time period, distance covered each minute, and the time to complete each 25-meter interval will be recorded. Standard encouragement using even, neutral tones was used for each participant according to the American Thoracic Society (ATS) guidelines [35] and as previously described for neuromuscular populations [36]. Percent-predicted distance on the six-minute walk test will be computed from normative values $[37,38]$. Fatigue will be determined by the decrement in distance walked from the first to sixth minute during the 6MWT [39].

\section{Secondary outcome measures}

\section{Exercise tolerance test}

Exercise tolerance testing provides an objective evaluation of overall fitness and is considered the best paradigm for assessing aerobic conditioning in diverse patient populations including neuromuscular disease [40]. Participants will undergo an exercise stress test using recumbent cycle ergometry pre- and post- exercise intervention at months $0,4,7,10,13$ and 19. The tests will be performed by a clinical exercise physiologist using an electronically-braked recumbent cycle ergometer (Lode Corival ${ }^{\mathrm{TM}}$, Groningen, Netherlands) using an individualized ramping protocol [40], maintaining a pedal cadence between $50-80 \mathrm{rpm}$. This will yield expected progressive workload increments of 5-10 Watts per minute as tolerated, following a 2 minute warm-up at 5 Watts. Participants will be continuously monitored for any discomfort or pain they may experience, and they will rate the exercise difficulty using a scale of perceived exertion using OMNI scale $\mathrm{TM}$ ratings of perceived exertion for cycling $[31,32]$. Maximal effort will be verified as volitional fatigue (OMNI Scale $\geq 8$, the inability to maintain pedal cadence at $50 \mathrm{rpm}$ for 10 or more seconds, and/or respiratory exchange ratio (RER; carbon dioxide production/oxygen uptake)>1.0) [40]. Standard ACSM exercise termination criteria will be applied [40].

Oxygen uptake, ventilatory volumes, and related variables will be measured during exercise using a ParvoMedics $^{\mathrm{TM}}$ metabolic cart (TrueOne $^{\mathrm{TM}}$ model 2400, Sandy, Utah). Measurements will end immediately following exercise. Using this method, peak oxygen uptake (VO2peak) will be identified as the highest oxygen uptake measured during exercise [41].

\section{Forced Vital Capacity (FVC)}

Pulmonary Function will be assessed by measuring forced expiratory vital capacity (FVC) as percent predicted for age and height [42]. Pulmonary function measures have been validated for use as an outcome measure in SMA in a previous study and the FVC was the most reliable measure [43].

\section{Hammersmith Functional Motor Scale, Expanded (HFMSE)}

The HFMSE is a 33-item scale designed for SMA type 2 and 3 patients; it represents minimal participant burden, requires only standard equipment, and is usually completed in less than 15 minutes. The HFMSE has good test-retest reliability and is correlated with clinical and physiological measures in SMA [44, 45]. Items are scored on a $0-2$ scale with a full score of 2 being normal without assistance, a partial score of 1 requiring a modified method but no assistance, and a zero score being unable to achieve the task without assistance. The sum of all items will be used as the outcome measure to evaluate motor function.

\section{0 meter walk/run}

A 10 meter walk / run test will be performed on all subjects at every visit. This test measures the time it takes a subject to walk or run 10 meters as fast as possible without compromising the subject's safety. In SMA, the 10 meter walk test correlated with knee extensor and flexor strength and discriminated between young and older ambulant patients [46]. The 10 meter walk/run test is an important adjunct to the 6MWT as it is a good measure 
of walking ability with minimal, if any, endurance demands.

\section{Timed Up and Go Test (TUG)}

The TUG test, which is a method to assess balance and mobility, measures the time it takes an individual to stand up from a chair, walk 3 meters, turn around, and sit down in the same chair [47]. In adult motor neuron disease, TUG scores are associated with standard functional and strength assessments, decline linearly over time, and predict falls [48]. In SMA, it demonstrates good test-retest reliability and is associated with standard clinical assessments [49].

\section{Muscle strength}

Manual muscle testing (MMT) augmented by handheld dynamometry (HHD) provides a comprehensive evaluation of muscle strength. In the present study, MMT will be performed on 28 muscle groups, 8 muscle groups on each leg and 6 muscle groups on each arm, including proximal and distal musculature, using the Medical Research Council (MRC) 10-point grading scale. In addition to recording the scores for each muscle group of the both legs and arms, the aggregate score of all four limb muscles as well both leg muscles and both arm muscles will be calculated. HHD will be performed on the knee flexors and extensors, elbow flexors and extensors, and shoulder abductor muscle groups bilaterally using a Micro FET ${ }^{\circledR}$ myometer. The best of 3 trials will be used for the analysis.

\section{Spatiotemporal gait measures}

Gait data will be collected with the GaitRite ${ }^{\mathrm{TM}}$ electronic walkway (GaitRITE, CIR Systems, Havertown, PA), which will be placed in the middle of the 25-meter walkway to collect steady state gait measures during the 6MWT. The computerized walkway is approximately 4.25 meters in length. Variables computed will include stride length and velocity for each stride in the first, third, and sixth minute during the 6MWT.

\section{Quantitative measure of physical activity}

We will measure habitual physical activity levels using the ActiGraph ${ }^{\mathrm{TM}}$ accelerometer (ActiLife 5, Pensacola, FL), worn on the waist and legs, over a 7 consecutive day period during each 3-month study segment. This device is a valid tool for measuring physical activity in children [50] and adults [51]. The ActiGraph is a triaxial accelerometer that measures acceleration in three planes; it is small, light, and unobtrusive. The acceleration is filtered by an analog band-pass filter and digitized by an 8-bit A/D converter at a sampling rate of 10 samples per second, storing data in user-defined intervals. This is an assessment of overall physical activity, so the accelerometer will be worn for 7 days, with one accelerometer on the waist and one on each ankle. To assist in interpreting the data, participants will also be given a log sheet to record specific times that the monitor was put on and taken off each day, as well as a record of sleeping times. The percentage of time spent sedentary will be calculated and used for analysis.

\section{Quality of life}

We will ask parents and patients to complete the "PedsQL ${ }^{\mathrm{TM}}$ Generic Quality of Life Inventory" and "Multidimensional Fatigue Scale," a proprietary test, to measure quality of life and perceived fatigue [52]. For patients 18 years and older, we will use the SF-36, a widely used health-related quality of life measure [53]. We will measure perceived fatigue in adult participants with a fatigue severity questionnaire that has been used in clinical research [54].

\section{Statistical analysis plan}

We will assess the efficacy of the exercise intervention based on change in distance walked during the $6 \mathrm{MWT}$ over a 6 month interval. The evaluations performed at month 1 will be used as the baseline assessment. Analysis will follow intent-to-treat principles; this approach will be followed regardless of subject dropout or un-blinding of the evaluating therapist. Data from a preliminary study of ambulatory subjects with SMA were used to estimate standard deviation for the outcome variable. From these preliminary assessments of patients followed longitudinally with the 6MWT, we have noted an average decline of $3.7 \%$ of the total distance walked (mean change $-3.7 \%$, absolute change $9.7 \%$, standard deviation $5.8 \%$ ) over a 6 month interval. Change in distance walked during the 6MWT has been used as the primary endpoint in studies designed to treat diseases with neuromuscular and pulmonary manifestations, including laronidase in mucopolysaccharidosis (MPS) type I [55] idursulfase in MPS II [56], and bosentan in primary pulmonary hypertension [57]. In these studies mean 6MWT distance in drug-treated patients relative to placebo-treated patients increased by $\sim 10 \%$. For the purposes of the power calculation, we will apply a clinically meaningful difference $(\delta)$ of $10 \%$. Standard deviation $(\sigma)$, based on preliminary data, will be 
defined as $5.8 \%$ for the purposes of the study. With 7 experimental subjects and 7 control subjects, $\alpha=0.05$ (two-sided), statistical power is 0.84 .

The mean difference in distance walked on the 6MWT over 6 months between the exercise and control group sample will be evaluated using an independent samples $t$-test.

The secondary analysis will include comparison between groups of changes in exercise tolerance (maximal O2 uptake and ventilatory threshold), HFMSE, 10 meter walk/run, quantified physical activity level (as measured using the Actigraph ${ }^{\mathrm{TM}}$ uniaxial accelerometer), kinematic gait assessment, FVC, Manual Muscle Testing (MMT) of 26 muscle groups augmented by Hand Held Dynamometry (HHD) of 10 standardized limb muscle groups, and quality of life outcomes over the first 6-month period. Repeated measures analysis of variance will be used to evaluate the effects of exercise on the primary and secondary outcomes for all participants over 18 and 12 months in the exercise and control groups respectively. For all analyses, differences will be considered statistically significant at alpha of $p \leq 0.05$.

\section{Participant characteristics}

Figure 2 shows the study participant flow. Fourteen participants, 3 females and 11 males, ages 10-48 years were enrolled and randomized between December 2009 and February 2013. Reasons for non-participation included not meeting eligibility requirements, enrollment in an alternative clinical trial, or disinterest. Participant characteristics are shown in Table 1. After randomization, the participants randomized to the control arm of the study were similar in age but had a somewhat younger age at symptom onset.

Table 2 describes the study outcome measures at baseline. At baseline, the control group walked a

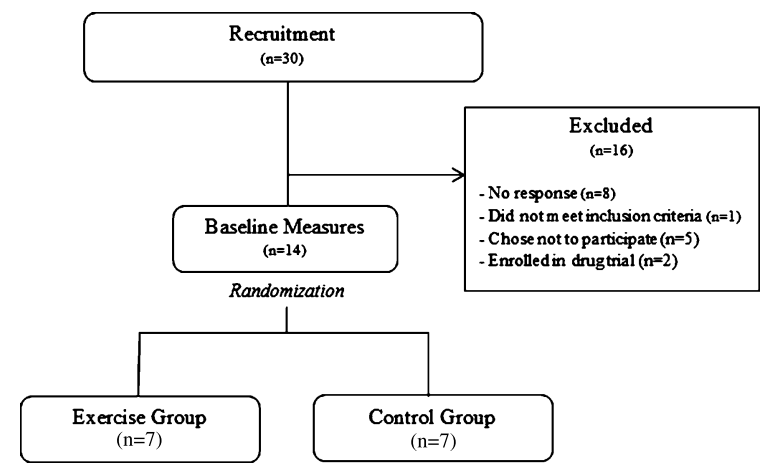

Fig. 2. Participant recruitment and randomization. shorter distance and, had more fatigue on the 6MWT. Control participants tended to be weaker as measured by strength assessments. All participants had normal pulmonary function and reduced exercise capacity. On average, the VO2 peak was $35.3 \%$ of the predicted value for age and gender. More than half of the participants $(64 \%)$ achieved a respiratory exchange ratio (RER) of $>1$; all terminated exercise due to an OMNI rating of $\geq 8$ and/or the inability to maintain a pedal rate of $50 \mathrm{rpm}$. This suggests that each participant gave a maximal effort.

Fatigue related gait changes during the 6MWT were measured using gait analysis in all participants. Velocity, stride length, and cadence decreased significantly across the first, third and sixth minutes $(p<0.001)$ (Fig. 3). Larger changes occurred during the first half of the 6MWT.

Both groups were insufficiently active, with a very small portion of time spent moving about, and $83.5 \%$ of time during waking hours spent in sedentary activities (e.g. sitting). Both groups participated in some physical activity of light, lifestyle and moderate intensities, while none engaged in any vigorous or very vigorous categories. Similar to knee flexor strength the control group was more sedentary.

Quality of life and fatigue measures were similar between groups. Adult participants evaluated their physical health as worse than their mental health (38.2, 54.0 - on a scale of 0-100) and reported moderate fatigue (4.8 on a scale of $0-7$ ). Child participants and their parents reported good quality of life overall (83, 86.5 - on a scale of $0-100$ ) while children reported slightly lower levels of fatigue than their parents $(85$, 74 - on a scale of 0-100). Because assessments of quality of life and fatigue are measured on different scales for adults and children, group comparisons were not performed.

\section{DISCUSSION}

The results of this prospective, single (examiner) blinded, randomized and controlled clinical trial of the effect of exercise should impact clinical practice by providing important guidance to the clinical management of SMA patients. In this study, the participants randomized to the control group had an average symptom onset that was somewhat earlier than the exercise group. Three of the 7 subjects in this group were termed Type $3 \mathrm{a}$ with symptom onset before age 3 years [3]. All 7 subjects in the exercise arm had symptom onset after age 3 years. There were subtle differences noted 
Table 1

Participant characteristics at baseline by study group

\begin{tabular}{|c|c|c|c|c|}
\hline \multirow[t]{2}{*}{ Subject characteristics } & \multicolumn{2}{|c|}{ Exercise group $(n=7)$} & \multicolumn{2}{|c|}{ Control group $(n=7)$} \\
\hline & Mean (SD) & Range & Mean (SD) & Range \\
\hline Age (years) & $27.0(14.6)$ & $10-43$ & $26.7(17.7)$ & $10-48$ \\
\hline Age Onset (years) & $\begin{array}{c}10.9(5.3) \\
n\end{array}$ & $5-19$ & $\begin{array}{c}6.4(5.4) \\
n\end{array}$ & $1-15$ \\
\hline Gender (male / female) & $6 / 1$ & & $5 / 2$ & \\
\hline SMA Tyре* & & & & \\
\hline $3 a$ & 0 & & 3 & \\
\hline $3 b$ & 7 & & 4 & \\
\hline
\end{tabular}

*3a - SMA type 3 patients symptomatic before the age of 3. $3 \mathrm{~b}$ - SMA type 3 patients symptomatic after the age of 3 .

Table 2

Outcome measurements at baseline by group assignment

\begin{tabular}{|c|c|c|c|c|}
\hline \multirow[t]{2}{*}{ Characteristics } & \multicolumn{2}{|c|}{ Exercise group $(n=7)$} & \multicolumn{2}{|c|}{ Control group $(n=7)$} \\
\hline & Mean (SD) & Range & Mean (SD) & Range \\
\hline 6MWT Distance (m) & $389.9(111.3)$ & $225-537$ & $331.6(133.0)$ & $289-498$ \\
\hline 6MWT (\% predicted) & $57.9(14.4)$ & $41.7-77.2$ & $50.0(21.9)$ & $20.0-80.6$ \\
\hline 6MWT (\% fatigue) & $22.0(10.9)$ & $2.7-36.0$ & $26.0(19.8)$ & $6.5-48.5$ \\
\hline TUG (s) & $12.4(7.03)$ & $5.41-24.5$ & $13.7(13.0)$ & $5.3-41.1$ \\
\hline 10-M Walk/Run (s) & $7.2(2.8)$ & $4.1-11.4$ & $7.8(3.0)$ & $4.0-13.8$ \\
\hline HFMSE (Total) & $53.4(8.9)$ & $44-63$ & $54.0(8.2)$ & $45-63$ \\
\hline MMT (Total) & $182.6(21.9)$ & $156-214$ & $185.0(33.4)$ & $122-209$ \\
\hline MMT (lower body) & $103.4(14.5)$ & $86-128$ & $106.3(22.3)$ & $66-121$ \\
\hline MMT (upper body) & $79.1(7.9)$ & $70-90$ & $78.7(14.2)$ & $56-92$ \\
\hline \multicolumn{5}{|l|}{ HHD (kg) } \\
\hline Shoulder AB (Right) & $4.0(1.4)$ & $2.4-6.0$ & $4.1(1.7)$ & $1.0-6.0$ \\
\hline Elbow Flexion (Right) & $13.0(5.9)$ & $5.8-23.7$ & $10.9(4.9)$ & $4.9-14.5$ \\
\hline Elbow Extension (Right) & $4.4(4.0)$ & $1.0-14.5$ & $4.0(2.3)$ & $0.8-6.2$ \\
\hline Knee Flexion (Right) & $6.8(1.3)$ & $4.6-12.3$ & $4.7(2.1)$ & $0.9-6.7$ \\
\hline Knee Extension (Right) & $4.1(4.5)$ & $0.4-6.5$ & $2.3(0.9)$ & $1.2-4.6$ \\
\hline $\mathrm{FVC}(\%$ predicted $)$ & $103.1(18.0)$ & $76-128$ & $103.0(14.1)$ & $83-123$ \\
\hline Exercise Capacity (mL/kg/min) & $14.4(4.4)$ & $8.6-19.5$ & $15.9(5.1)$ & $9.7-25.1$ \\
\hline \multirow[t]{2}{*}{$\%$ Predicted Exercise Capacity } & $34.4(6.8)$ & $25.9-44.0$ & $41.9(12.2)$ & $30.7-61.7$ \\
\hline & \multicolumn{2}{|c|}{ Exercise group $(n=5)$} & \multicolumn{2}{|c|}{ Control group $(n=6)$} \\
\hline Physical Activity - \% Sedentary & $76.9(0.1)$ & $71.0-85.3$ & $90.1(0.1)$ & $80.4-100.0$ \\
\hline
\end{tabular}

6MWT $=$ Six Minute Walk Test, SMA-FC $=$ SMA Functional Composite Score, TUG $=$ Timed Up and Go Test, HFMSE $=$ Hammersmith Functional Motor Scale, Expanded, MMT = Manual Muscle Test, HHD = Hand-held Dynamometry, FVC = Forced Vital Capacity.

in walking ability and strength between the groups. Interestingly, knee flexor strength was more impaired and the participants were more sedentary in the control group. It is not known if these differences will influence responsiveness to exercise. A larger study will be necessary to identify which SMA characteristics are most amenable to treatment.

Exercise testing provides an objective measure of functional capacity and overall fitness and is considered the best measure of aerobic conditioning in diverse patient populations including neuromuscular disease [29]. Ambulant SMA patients have reduced muscle strength and cardiorespiratory fitness, consistent with severe deconditioning, and mostly normal pulmonary function as determined by forced vital capacity. Exercise capacity measured by peak oxygen uptake (VO2

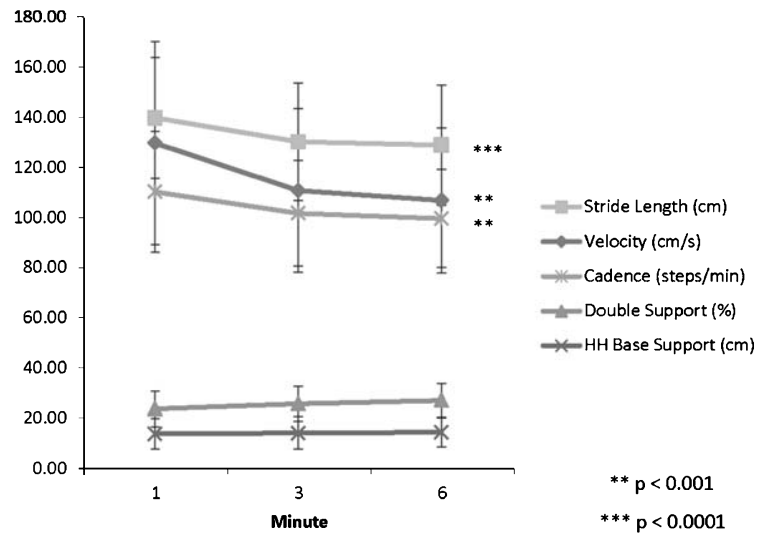

Fig. 3. Baseline gait parameters for all participants at minutes 1,3 , and 6 during the $6 \mathrm{MWT}$. 
Table 3

Participant quality of life and perceived fatigue at baseline by group assignment

\begin{tabular}{cccc}
\hline & & $\begin{array}{c}\text { Exercise } \\
\text { group }(n=5)\end{array}$ & $\begin{array}{c}\text { Control } \\
\text { group }(n=4)\end{array}$ \\
\cline { 3 - 3 } & Outcome Variable & Mean (SD) & Mean (SD) \\
\hline PDULT & SF-36 & & \\
& Mental Health & $57.7(7.3)$ & $38.7(4.8)$ \\
FSS & $4.61(1.7)$ & $5.0(.85)$ \\
\hline CHILD & Neuromuscular (PedsQL) & & Control \\
& Child & $81(2.8)$ & $85(12.9)$ \\
& Parent & $84(17.7)$ & $89(4.9)$ \\
& Fatigue (PedsQL) & & \\
Child & $93(6.5)$ & $77(17.3)$ \\
& Parent & $64(14.0)$ & $84(11.9)$ \\
\hline
\end{tabular}

SF-36 = Short Form (36) Health Survey, FSS = Fatigue Severity Scale, PedsQL = Pediatric Quality of Life Inventory.

max) appears to be lower in ambulatory SMA patients compared to other myopathic [14, 15, 17-19, 58] and denervating [59-62] neuromuscular disorders, which may represent an important clue to pathophysiological differences. Evidence suggests that mitochondrial depletion in SMA [63] may be causative and will warrant further exploration. The number and functionality of mitochondria will affect the muscle function, and thereby affect physical functioning and overall health [13].

The considerable sedentary behavior and resulting deconditioning experienced by SMA patients likely contributes to the depressed cardiorespiratory capacity observed, which may reflect the consequences of mitochondrial depletion [13]. The potential effect of deconditioning on daily functioning is further demonstrated by the impairments demonstrated on the functional assessments. Our participants walked $54 \%$ of what is expected on the 6MWT, and exhibited impaired muscle strength in nearly all of the muscle groups tested. Fatigue during the 6MWT likely represents peripheral and central dysfunction of neurotransmission that is unique to the pathophysiology of SMA; and this also may have an impact on overall fitness since aerobic fitness represents the integrated function of many body systems, including the cardiorespiratory and neuromuscular systems.

The results of the pulmonary function tests combined with the results of the exercise testing show that the pulmonary system does not limit exercise in these participants with SMA, similar to healthy individuals. The ability of many of the participants to achieve a respiratory exchange ratio (RER) of 1.0 or greater is consistent with reaching the limits of cardiorespiratory system function [29]. Neuromuscular factors do not limit exercise capacity at least in some SMA patients. However, other patients experienced limiting muscular fatigue as demonstrated by their inability to maintain the requisite pedaling rate in the presence of an RER less than 1.0. The absence of any adverse effects during the baseline testing show, importantly, that exercise testing is feasible. Furthermore, pulmonary function testing complements functional measures that are designed to evaluate exercise interventions in ambulatory SMA patients.

To our knowledge, this will be the first randomized, controlled clinical trial of exercise training following national recommendations for exercise in SMA. It will advise and inform future clinical practice and research study design. Moreover, the results of this study will provide mechanistic information on the changes underlying any observed improvement in exercise performance and may improve our understanding of the processes underlying weakness and fatigue in SMA.

\section{ACKNOWLEDGMENTS}

This study is supported by the Department of Defense; USAMRAA Grant/Cooperative award number: 09131005 (W81XWH-10-1-0127). The sponsors had no role in the conduct of this study. We gratefully acknowledge the research participants and their families for their generous gift of time and effort.

\section{CONFLICT OF INTEREST}

The authors have no conflict of interest to report.

\section{REFERENCES}

[1] Lefebvre, S., Burglen, L., Reboullet, S., Clermont, O., Burlet, P., Viollet, L., et al. Identification and characterization of a spinal muscular atrophy-determining gene. Cell. 1995; 80(1): $155-165$.

[2] Munsat, T. L., Skerry, L., Korf, B., Pober, B., Schapira, Y., Gascon, G. G., et al. Phenotypic heterogeneity of spinal muscular atrophy mapping to chromosome 5q11.2-13.3 (SMA 5q). Neurology. 1990; 40(12): 1831-1836.

[3] Zerres, K., Rudnik-Schoneborn, S. Natural history in proximal spinal muscular atrophy. Clinical analysis of 445 patients and suggestions for a modification of existing classifications. Arch Neurol. 1995; 52(5): 518-523.

[4] Garber, C. E., Blissmer, B., Deschenes, M. R., Franklin, B. A., Lamonte, M. J., Lee, I. M. et al. American College of Sports Medicine position stand. Quantity and quality of exercise for developing and maintaining cardiorespiratory, musculoskeletal, and neuromotor fitness in apparently healthy adults: Guidance for prescribing exercise. Med Sci Sports Exerc. 2011; 43(7): 1334-1359. 
[5] U.S. Department of Health and Human Services. 2008 Physical Activity Guidelines for Americans. [[Internet]]. Washington., D.C.: U.S. Department of Health and Human Services.; 2008 [cited 2008. Available from: http://www. health.gov/paguidelines/pdf/paguide.pdf

[6] Keeton, V. F., Kennedy, C. Update on physical activity including special needs populations. Current opinion in pediatrics. 2009; 21(2): 262-268.

[7] Nelson, M. E., Rejeski, W. J., Blair, S. N., Duncan, P. W., Judge JO., King AC., et al. Physical activity and public health in older adults: Recommendation from the American College of Sports Medicine and the American Heart Association. Med Sci Sports Exerc. 2007; 39(8): 1435-1445.

[8] Lubans, D. R., Morgan, P. J., Cliff, D. P., Barnett, L. M., Okely, A. D. Fundamental movement skills in children and adolescents: Review of associated health benefits. Sports medicine (Auckland., NZ). 2010; 40(12): 1019-1035.

[9] Rimmer, J. H., Schiller, W., Chen, M. D. Effects of disability-associated low energy expenditure deconditioning syndrome. Exercise and sport sciences reviews. 2012; 40(1): 22-29.

[10] Phillips, S. M. Physiologic and molecular bases of muscle hypertrophy and atrophy: Impact of resistance exercise on human skeletal muscle (protein and exercise dose effects). Applied physiology., nutrition., and metabolism=Physiologie appliquee., nutrition et metabolisme. 2009; 34(3): 403-410.

[11] Urso, M. L. Disuse atrophy of human skeletal muscle: Cell signaling and potential interventions. Medicine and science in sports and exercise. 2009; 41(10): 1860-1868.

[12] Hood, D. A. Mechanisms of exercise-induced mitochondrial biogenesis in skeletal muscle. Appl Physiol Nutr Metab. 2009; 34(3): 465-472.

[13] Russell, A. P., Foletta, V. C., Snow, R. J., Wadley, G. D. Skeletal muscle mitochondria: A major player in exercise, health and disease. Biochimica et biophysica acta, 2013.

[14] Jeppesen, T. D., Schwartz, M., Olsen, D. B., Wibrand, F., Krag, T., Duno, M., et al. Aerobic training is safe and improves exercise capacity in patients with mitochondrial myopathy. Brain. 2006; 129(Pt 12): 3402-3412.

[15] Sveen, M. L., Jeppesen, T., Hauerslev, S., Kober, L., Krag, T. O., Vissing, J. Endurance training improves fitness and strength in patients with Becker muscular dystrophy. Brain. 2008; p. 2824-2831.

[16] Haller, R. G., Wyrick, P., Taivassalo, T., Vissing, J. Aerobic conditioning: An effective therapy in McArdles' disease. Ann Neurol. 2006; 59(6): 922-928.

[17] Olsen, D. B., Orngreen, M. C., Vissing, J. Aerobic training improves exercise performance in facioscapulohumeral muscular dystrophy. Neurology. 2005; 64(6): 1064-1066.

[18] Sveen, M. L., Jeppesen, T., Hauerslev, S., al. E. Endurance training: An effective and safe treatment for patients with LGMD2I. Neurology. 2007; 68: 59-61.

[19] Johnson, L., Collier, K., Edwards, D., Philippe, D., Eastwood, P., Walters, S., et al. Improvement in Aerobic Capacity After an Exercise Program in Sporadic Inclusion Body Myositis. Journal of Clinical Neuromuscular Disease. 2009; 10(1): 178181.

[20] Sveen, M. L., Andersen, S. P., Ingelsrud, L. H., Blichter, S., Olsen, N. E., Jonck, S., et al. Resistance training in patients with limb-girdle and becker muscular dystrophies. Muscle \& nerve. 2013 ; 47(2): 163-169.

[21] Bello-Haas, V. D., Florence, J. M., Kloos, A. D., Scheirbecker, J., Lopate, G., Hayes, S. M., et al. A randomized controlled trial of resistance exercise in individuals with ALS. Neurology. 2007; 68(23): 2003-2007.
[22] Dawes, H., Korpershoek, N., Freebody, J., Elsworth, C., van Tintelen, N., Wade, D. T., et al. A pilot randomised controlled trial of a home-based exercise programme aimed at improving endurance and function in adults with neuromuscular disorders. J Neurol Neurosurg Psychiatry. 2006; 77(8): 959-962.

[23] Grondard, C., Biondi, O., Armand, A. S., Lecolle, S., Della Gaspera, B., Pariset, C., et al. Regular exercise prolongs survival in a type 2 spinal muscular atrophy model mouse. J Neurosci. 2005; 25(33): 7615-7622.

[24] Biondi, O., Grondard, C., Lecolle, S., Deforges, S., Pariset, C., Lopes, P., et al. Exercise-induced activation of NMDA receptor promotes motor unit development and survival in a type 2 spinal muscular atrophy model mouse. J Neurosci. 2008; 28(4): 953-962.

[25] Granata, C., Colombari, M., Ballestrazzi, A., Bombardi, F., Granata, L., Merlini, L. Isokinetic Test and Exercise in Spinal Muscular Atrophy (SMA). IEEE Engineering in Medicine and Biology Society., 11th Annual International Conference, 1989.

[26] Salem, Y., Gropack, S. J. Aquatic therapy for a child with type III spinal muscular atrophy: A case report. Phys Occup Ther Pediatr. 2010; 30(4): 313-324.

[27] Cup, E. H., Pieterse, A. J., Ten Broek-Pastoor, J. M., Munneke, M., van Engelen, B. G., Hendricks, H. T., et al. Exercise therapy and other types of physical therapy for patients with neuromuscular diseases: A systematic review. Arch Phys Med Rehabil. 2007; 88(11): 1452-1464.

[28] Kilmer, D. D., Aitkens, S. G., Wright, N. C., McCrory, M. A. Response to high-intensity eccentric muscle contractions in persons with myopathic disease. Muscle Nerve 2001; 24 : 1181-1187.

[29] ACSM' guidelines for exercise testing and prescription. 9th ed ed. Pescatello, L. S., American College of Sports, M., editors. Philadelphia: Wolters Kluwer Health/Lippincott Williams \& Wilkins; 2013 c2013.

[30] American College of Sports Medicine. Guidelines for Exercise Testing and Prescription. 5th ed. Kenney, W. L., editor. Baltimore: Williams \& Wilkins; 1995; 269-87.

[31] Lagally, K. M., Robertson, R. J. Construct validity of the OMNI resistance exercise scale. J Strength Cond Res. 2006; 20(2): 252-256.

[32] Robertson, R. J., Goss, F. L., Dube, J., Rutkowski, J., Dupain, M., Brennan, C., et al. Validation of the adult OMNI scale of perceived exertion for cycle ergometer exercise. Med Sci Sports Exerc. 2004; 36(1): 102-108. PubMed PMID: 14707775. Epub 2004/01/07. eng.

[33] Deymeer, F., Serdaroglu, P., Poda, M., Gulsen-Parman, Y., Ozcelik, T., Ozdemir, C. Segmental distribution of muscle weakness in SMA III: Implications for deterioration in muscle strength with time. Neuromuscul Disord. 1997; 7(8): 521-528.

[34] American College of Sports Medicine position stand. Progression models in resistance training for healthy adults. Med Sci Sports Exerc. 2009; 41(3): 687-708.

[35] ATS statement: Guidelines for the six-minute walk test. Am J Respir Crit Care Med. 2002; 166(1): 111-117.

[36] Montes, J., Blumenschine, M., Dunaway, S., Alter, A. S., Engelstad, K., Rao, A. K., et al. Weakness and fatigue in diverse neuromuscular diseases. J Child Neurol. 2013; 28(10): 1277-1283.

[37] Geiger, R., Strasak, A., Treml, B., Gasser, K., Kleinsasser, A., Fischer, V., et al. Six-minute walk test in children and adolescents. J Pediatr. 2007; 150(4): 395-399.

[38] Gibbons, W. J., Fruchter, N., Sloan, S., Levy, R. D. Reference values for a multiple repetition 6-minute walk test in healthy 
adults older than 20 years. J Cardiopulm Rehabil. 2001; 21(2): 87-93.

[39] Montes, J., McDermott, M. P., Martens, W. B., Dunaway, S., Glanzman, A. M., Riley, S., et al. Six-Minute Walk Test demonstrates motor fatigue in spinal muscular atrophy. Neurology. 2010; 74(10): 833-838.

[40] ACSM' guidelines for exercise testing and prescription. 9th ed ed. Pescatello, L. S., American College of Sports, M., editors. Philadelphia: Wolters Kluwer Health/Lippincott Williams \& Wilkins; 2010 c2013.

[41] Wasserman, K., Stringer, W. W., Casaburi, R., Koike, A., Cooper, C. B. Determination of the anaerobic threshold by gas exchange: Biochemical considerations., methodology and physiological effects. Z Kardiol. 1994; 83(Suppl 3): 1-12.

[42] Wang, X., Dockery, D. W., Wypij, D., Fay, M. E., Ferris, B. G., Jr. Pulmonary function between 6 and 18 years of age. Pediatr Pulmonol. 1993; 15(2): 75-88.

[43] Iannaccone, S. T. Outcome measures for pediatric spinal muscular atrophy. Arch Neurol. 2002; 59(9): 1445-1450.

[44] O'Hagen, J. M., Glanzman, A. M., McDermott, M. P., Ryan, P. A., Flickinger, J., Quigley, J., et al. An expanded version of the Hammersmith Functional Motor Scale for SMA II and III patients. Neuromuscul Disord. 2007; 17(9-10): 693-697.

[45] Glanzman, A. M., O'Hagen, J. M., McDermott, M. P., Martens, W. B., Flickinger, J., Riley, S., et al. Validation of the Expanded Hammersmith Functional Motor Scale in spinal muscular atrophy type II and III. J Child Neurol. 2011;26(12): 1499-1507.

[46] Merlini, L., Bertini, E., Minetti, C., Mongini, T., Morandi, L., Angelini, C., et al. Motor function-muscle strength relationship in spinal muscular atrophy. Muscle Nerve. 2004; 29(4): $548-552$.

[47] Podsiadlo, D., Richardson, S. The timed "Up \& Go": A test of basic functional mobility for frail elderly persons. Journal of the American Geriatrics Society. 1991; 39(2): 142-148.

[48] Montes, J., Cheng, B., Diamond, B., Doorish, C., Mitsumoto H., Gordon, P. H. The Timed Up and Go test: Predicting falls in ALS. Amyotroph Lateral Scler. 2007; 8(5): 292-295.

[49] Dunaway, S., Montes, J., Garber, C. E., Carr, B., Kramer, S. S., Kamil-Rosenberg, S., et al. Performance of the timed "up \& go" test in spinal muscular atrophy. Muscle \& nerve. 2013. PubMed PMID: 24375426. Epub 2014/01/01.

[50] Pfeiffer, K. A., McIver, K. L., Dowda, M., Almeida, M. J., Pate, R. R. Validation and calibration of the Actical accelerometer in preschool children. Med Sci Sports Exerc. 2006; 38(1): 152-157.

[51] Esliger, D. W., Probert, A., Gorber, S. C., Bryan, S., Laviolette, M., Tremblay, M. S. Validity of the Actical accelerometer step-count function. Med Sci Sports Exerc. 2007; 39(7): 1200-1204.

[52] Varni, J. W., Seid, M., Rode, C. A. The PedsQL: Measurement model for the pediatric quality of life inventory. Medical care. 1999; 37(2): 126-139.

[53] Ware, J., Jr., Kosinski, M., Keller, S. D. A 12-Item Short-Form Health Survey: Construction of scales and preliminary tests of reliability and validity. Medical care. 1996; 34(3): 220-233.

[54] Krupp, L. B., LaRocca, N. G., Muir-Nash, J., Steinberg, A. D. The fatigue severity scale. Application to patients with multiple sclerosis and systemic lupus erythematosus. Arch Neurol. 1989; 46(10): 1121-1123.

[55] Wraith, J. E., Clarke, L. A., Beck, M., et al. Enzyme replacement therapy for mucopolysaccharidosis I: A randomized., double-blinded., placebo-controlled., multinational study of recombinant human alpha-L-iduronidase (laronidase). J Pediatr 2004; 144: 581-588.

[56] Muenzer, J., Wraith, J. E., Beck, M., et al. A phase II/III clinical study of enzyme replacement therapy with idursulfase in mucopolysaccharidosis II (Hunter syndrome). Genet Med. 2006; 8: 465-473.

[57] Rubin, L. J., Badesch, D. B., Barst, R. J., et al. Bosentan therapy for pulmonary arterial hypertension. N Engl J Med. 2002; 346: 896-903.

[58] Orngreen, M. C., Olsen, D. B., Vissing, J. Aerobic training in patients with myotonic dystrophy type 1. Ann Neurol. 2005; 57(5): 754-757.

[59] Preisler, N., Andersen, G., Thogersen, F., Crone, C., Jeppesen, T. D., Wibrand, F., et al. Effect of aerobic training in patients with spinal and bulbar muscular atrophy (Kennedy disease). Neurology. 2009; 72(4): 317-323.

[60] Jones, D., Speier, J., Canine, K., Owen, R., Stull, A. Cardiorespiratory Responses to Aerobic Training by Patients With Postpoliomyelitis Sequelae. JAMA: The journal of the American Medical Association. 1989; 261(22): 3255-3258.

[61] Mhandi, L., Millet, G., Calmels, P., Richard, A., Oullion, R., Gautheron, V., et al. Benefits of interval-training on fatigue and funtional capacities in Charcot-Marie-Tooth disease. Muscle \& Nerve. 2008; 37: 601-610.

[62] Sanjak, M., Paulson, D., Sufit, R., Reddan, W., Beaulieu, D., Erickson, L., et al. Physiologic and metabolic response to progressive and prolonged exercise in amyotrophic lateral sclerosis. Neurology. 1987; 37: 1217-1220.

[63] Berger, A., Mayr, J. A., Meierhofer, D., Fotschl, U., Bittner, R., Budka, H., et al. Severe depletion of mitochondrial DNA in spinal muscular atrophy. Acta Neuropathol. 2003; 105(3): 245-251. 